\title{
Laser de diodo no tratamento da retinopatia da prematuridade
}

\author{
Diode laser in the treatment of retinopathy of prematurity
}

Josilene de Carvalfio Soares Liarth $^{1}$
João Orlando Ribeiro Gonçalves
Ednaldo Atem Gonçalves
Eridê Sousa Meneses $^{3}$
Fábio Martins $\quad$ Soares

Serviço de Retina e Vítreo do Hospital Getúlio Vargas; Centro de Ciências da Saúde - Universidade Federal do Piauí; Instituto de Olhos do Piauí - Teresina (PI). ${ }^{1}$ Médica oftalmologista do Hospital Getúlio Vargas.

${ }_{2}^{2}$ Professor titular de Oftalmologia da Universidade Federal do Piaú e chefe da Clínica Oftalmológica do Hospital Getúlio Vargas.

${ }^{3}$ Professor-assistente de Oftalmologia da Universidade Federal do Piauí.

${ }^{4}$ Médica oftalmologista do Hospital Getúlio Vargas.

${ }^{5}$ Residente do $2^{\mathrm{a}}$ ano do Serviço de Oftalmologia da

Universidade Federal do Piauí - Hospital Getúlio Vargas.

Endereco para correspondência: R. Senador Cândido Ferraz, 1945, apto 401 - Teresina (PI) CEP 64049-250.

\section{INTRODUÇÃO}

A retinopatia da prematuridade (R.P.) é uma doença vasoproliferativa que acomete a retina de crianças prematuras, principalmente as com peso de nascimento menor que 1250 gramas e com idade gestacional (IG) menor que 31 semanas, sendo causa importante de cegueira na infância.

A classificação internacional da R.P..$^{(1-2)}$ descreve cinco estágios da doença: estágio 1 - linha de demarcação; estágio 2 - crista retiniana; estágio 3 - crista retiniana com proliferação fibrovascular extra-retiniana; estágio 4 - descolamento da retina, A- extrafoveal e B-incluindo a fóvea; e estágio 5 - descolamento total da retina.

O tratamento da R.P. é feito de acordo com o estágio da doença. Nos estágios 1 e 2, a conduta é expectante. No estágio $3+$ plus (tortuosidade e ingurgitamento vascular retiniano) na zona 1 ou 2 , com extensão de cinco ou mais horas contíguas ou oito horas cumulativas de neovascularização, perfazendo $240^{\circ}$ (estágio 3 limiar) há indicação para crioablação ou laserablação retiniana. Nos estágios 4 e 5 estão indicados procedimentos mais agressivos como introflexão escleral, vitrectomia ou outros, conforme a necessidade. $\mathrm{O}$ tratamento aplicado, bem como a anestesia para a realização do mesmo, podem levar a complicações oculares e/ou sistêmicas. 
Nesse contexto, procurou-se determinar a eficácia do laser de diodo e suas complicações no tratamento da R.P. no estágio 3 limiar.

\section{MÉTODOS}

Examinaram-se com oftalmoscopia binocular indireta (O.B.I.), 348 crianças prematuras, encaminhadas pelo setor de neonatologia da Maternidade Dona Evangelina Rosa, ao Serviço de Retina e Vítreo da Clínica Oftalmológica do Hospital Getúlio Vargas (H.G.V.) e Instituto de Olhos do Piauí (I.O.P.), em Teresina-Piauí, no período de julho de 1989 a março de 1999. Os critérios utilizados para o exame foram: prematuridade com peso $\leq 1500 \mathrm{~g}$ ou $\mathrm{IG} \leq 32$ semanas e prematuridade com peso $>1500 \mathrm{~g}$ ou $\mathrm{IG}>32$ semanas associado a oxigenoterapia, transfusão sanguínea, infecção ou antibioticoterapia. As crianças foram examinadas entre 4 e 6 semanas de vida.

Cento e cinquenta e duas crianças apresentaram R.P. ao primeiro exame, sendo: $5,26 \%$ na forma regressiva; $49,34 \%$ no estágio $1 ; 8,55 \%$ no estágio $2 ; 11,18 \%$ no estágio $3 ; 3,29 \%$ no estágio $4 ; 21,05 \%$ no estágio 5 e $1,32 \%$ na zona 1 , muito posterior. Dentre os pacientes nos estágios 1 e 2, oito evoluíram para o estágio 3 da doença.

Submeteram-se as crianças com diagnóstico de R.P. no estágio 3 limiar à laserablação retiniana com laser de diodo (Iris medical - oculight) através de O.B.I., com marcas espaçadas, no centro cirúrgico, após dilatação pupilar com fenilefrina $2,5 \%$ e tropicamida $0,5 \% 3 \mathrm{x}$ cada, sob anestesia geral com halotano. Apenas uma criança, foi tratada mais de uma vez, 16 meses após o primeiro procedimento. $\mathrm{O}$ tempo médio de observação dos pacientes foi de 12 meses.

\section{RESULTADOS}

Vinte crianças foram submetidas a laserablação retiniana, no total de 38 olhos, sendo 11 crianças do sexo feminino e nove do sexo masculino (Quadro 1). Uma criança necessitou de laser em apenas um olho e outra teve o procedimento interrompido antes do tratamento do segundo olho.

O peso ao nascimento variou de $820 \mathrm{~g}$ a $1470 \mathrm{~g}$ com peso

\begin{tabular}{|c|c|}
\hline \multicolumn{2}{|c|}{$\begin{array}{l}\text { Quadro 1. Distribuição dos pacientes com retinopatia da prema- } \\
\text { turidade, submetidos a Laser de diodo na clínica oftalmológica do } \\
\text { Hospital Getúlio Vargas, período julho de1989 a março de 1999, } \\
\text { Teresina-PI }\end{array}$} \\
\hline PARÂMETRO & $\mathbf{N}^{\circ}$ \\
\hline Pacientes tratados & 20 \\
\hline Olhos tratados & 38 \\
\hline Sexo (Masculino / feminino) & $9 / 11$ \\
\hline Peso médio ao nascer & $1.144 \mathrm{~g}$ \\
\hline Idade gestacional média & 30 sem \\
\hline Idade gestacional pós-conceptual média & 41 sem \\
\hline Marcas de laser (média) & 370 \\
\hline
\end{tabular}

médio de $1144 \mathrm{~g}$; a idade gestacional de 26 a 34 semanas,com idade gestacional média de 30 semanas e a idade gestacional pós-conceptual na época do tratamento de 34 a 46 semanas, com média de 41 semanas.

O número de marcas de laser por olho, foi em média 370 , variando de 71 a 1096, algumas crianças tiveram o procedimento suspenso e o tratamento incompleto, devido apnéia.

As complicações gerais que ocorreram foram paradas respiratórias, em cinco crianças $(25,0 \%)$.Em duas crianças a apnéia ocorreu durante a indução anestésica. As demais crianças apresentaram apnéia durante a laserablação. Algumas apresentaram mais de uma apnéia. Não foram observadas complicações oculares relacionadas ao tratamento com o laser de diodo.

Doze crianças $(80,0 \%)$ tiveram regressão da retinopatia, e três $(20,0 \%)$ evoluíram para o estágio 5 (Tabela 2). Cinco crianças não retornaram para controle sendo excluídas do estudo quanto aos resultados do laser. As crianças que evoluiram para descolamento total da retina tinham peso ao nascimento e idade gestacional respectivamente: $1100 \mathrm{~g} / 27$ semanas, $1470 \mathrm{~g} / 32$ semanas e $1420 \mathrm{~g} / 29$ semanas.

\section{DISCUSSÃO}

A ablação da retina avascular tem reduzido significativamente a morbidade da R.P., em estágio avançado. Esta ablação foi primeiramente descrita com o xenônio em $1968^{(3)}$. Posteriormente o laser de argônio foi usado para tratar a doença. Em 1981, foi introduzido o laser através da oftalmoscopia indireta. As retinopatias que podem ser tratadas com o laser de argônio podem ser tratadas com o laser de diodo. O estudo de Benner et al. ${ }^{(4)}$ comparou a fotocoagulação com o laser de argônio verde, kriptônio vermelho e o laser de diodo através da oftalmoscopia indireta em olhos de coelhos e demonstrou a variação na intensidade e diâmetro da marca com os três tipos de laser.

O laser de diodo na fotocoagulação retiniana, por apresentar comprimento de onda grande $(810 \mathrm{~nm})$, pode ser utilizado em casos de opacificação cristaliniana moderada e hemorragia vítrea leve, pois apresenta boa transmissão nesses meios, ao contrário do laser de argônio azul-verde e verde ${ }^{(5)}$. Neste estudo, uma criança com R.P. na zona 1 muito posterior, apresentou dilatação venosa e hemorragia retiniana em um olho, 16 meses após o primeiro procedimento, sendo realizado nova laser ablação retiniana e evoluído bem após o tratamento.

Tabela 2. Distribuição dos pacientes com retinopatia da prematuridade, no estágio 3 limiar segundo os resultados do tratamento com laser de diodo indireto na clínica oftalmológica do Hospital Getúlio Vargas, período julho de1989 a março de 1999, Teresina-PI
RESULTADO

Favorável

Não-favorável

TOTAL

\begin{tabular}{cc|}
$\mathbf{N}^{\circ}$ & $\%$ \\
12 & 80 \\
03 & 20 \\
15 & 100 \\
\hline
\end{tabular}


Os resultados iniciais do uso da crioterapia transescleral para tratamento da R.P. em estágios avançados, em 1970, foram desfavoráveis ${ }^{(3)}$. Porém, o seu uso clínico foi aceito após sua utilização no Japão, em $1972^{(6-7)}$. O estudo multicêntrico de crioterapia para tratamento da R.P. ${ }^{(6)}$ demonstrou que a evolução desfavorável foi significantemente menos freqüente em olhos tratados com a crioterapia transescleral retiniana do que nos olhos não tratados, demonstrando a eficácia do tratamento na redução da progressão da doença para descolamento de retina ou tecido retrocristaliniano.

Vários estudos ${ }^{(8-10)}$ compararam a eficácia da crioterapia com a fotocoagulação com laser na R.P. e concluíram que estas técnicas são equivalentes na eficácia, embora a laserterapia apresente menos complicações per e pós-operatórias. Neste estudo não foi comparado laserablação com crioablação.

No presente trabalho, o laser de diodo foi eficaz em $80,0 \%$ dos casos (12 pacientes) - 23 olhos - com regressão completa da retinopatia. No estudo de Moraes et al. ${ }^{(10)}$ todas as crianças tratadas com laser de diodo indireto apresentaram regressão da doença.

O número de marcas de laser por olho, variou de acordo com a necessidade, com uma média de 370 marcas por olho. A criança que necessitou de um maior número de disparos (1096 disparos no olho direito e 1049 , no esquerdo) foi um dos casos de R.P. na zona 1 muito posterior. A mesma, apesar do tratamento, evoluiu para o estágio 5.

A primeira das duas crianças com RP estágio 3 limiar na zona 1 muito posterior, evoluiu bem após a laserablação retiniana; porém, 16 meses após o tratamento, apresentou hemorragia retiniana e dilatação venosa em um olho sendo necessária a repetição do laser neste olho, com regressão da doença após o mesmo. A segunda, como citado no parágrafo anterior, apresentou resultado insatisfatório, com evolução para descolamento total da retina. No estudo de Capone et al. ${ }^{(11)}$, em que foi usado o laser de diodo no tratamento da RP estágio 3 limiar na zona $1,16,7 \%$ dos olhos desenvolveram descolamento de retina.

McNamara et al. ${ }^{(12)}$ relacionaram as complicações da fotocoagulação com laser na R.P.: edema corneano, lesão iriana, lesão no cristalino, hemorragias retiniana, pré-retiniana ou vítrea, hemorragia coroidal, neovascularização de coróide, fotocoagulação acidental da fóvea, formação de membrana pré retiniana e descolamento de retina tardio. Neste trabalho não foram observadas tais complicações oculares. Entretanto, cinco crianças apresentaram apnéia possivelmente relacionada à anestesia e, em algumas delas, o procedimento teve que ser suspenso, em uma inclusive, a complicação ocorreu durante o tratamento do primeiro olho, não tendo sido realizado no segundo olho. Esta criança não retornou para controle.

\section{CONCLUSÕES}

Neste grupo de crianças, o laser de diodo foi eficaz em $80,0 \%$ dos pacientes tratados no estágio 3 limiar da RP. As complicações encontradas (cinco casos de apnéia) se relacionaram possivelmente à anestesia geral.

SUMMAR $Y$

Purpose: To determine the efficacy of the diode laser and its complications in treating retinopathy of prematurity, at stage 3, threshold. Methods: One hundred and fifty-two children, out of 348 premature infants examined at the Ophthalmologic Clinic of the Getúlio Vargas Hospital and Instituto de Olhos do Piauí, in Teresina - Piauí, from July '89 to March '99, had retinopathy of prematurity. Those with retinopathy of prematurity at stage 3 , threshold, were submitted to application of diode indirect laser to the retina. Results: Twenty children (38 eyes) received diode laser. Twelve patients showed regression of retinopathy and three children developed stage 5 . No ocular complications were observed. Five children had respiratory failure due to anesthesia. Conclusions: The diode laser treatment was successful in $80.0 \%$ of the patients. The complications were possibly related to the general anesthesia.

Keywords: Retinopathy of prematurity/therapy; Light coagulation; Lasers/therapeutic use; Infant, newborn; Infant, premature

\section{REFERÊNCIAS}

1. An international classification of retinopathy of prematurity.Committee for the classification of retinopathy of prematurity. Arch Ophthalmol 1984;102: 1130-4.

2. An international classification of retinopathy of prematurity. The classification of retinal dettachment The International Committee for the Classification of the Late Stages of Retinopathy of Prematurity. Arch Ophthal 1987;105: 906-12.

3. Nagata M, Kobayashi Y, Fukuda H, apud Benner JD. Photocoagulation with the laser indirect ophthalmoscope for retinopathy of prematurity. Semin Ophtalmol 1992;7:177-81.

4. Benner JD, Huang M, Morse LS, Hielmeland LM, Landers MB. Comparison of photocoagulation with the argon, krypton, and diode laser indirect ophthalmoscopes in rabbit eyes. Ophthalmology 1992;99:1544-63.

5. Gonçalves JCM. Laser de diodo. Arq Bras Oftalmol 1996;59:103-4.

6. Multicenter trial of cryotherapy for retinopathy of prematurity - preliminary results. Cryotherapy for Retinopathy of Prematurity Cooperative Group. Arch Ophthalmol 1988;106:471-9.

7. Yamashita Y. Study on retinopathy of prematurity (III). Cryocautery for retinopathy of prematurity. Jpn J Clin Ophthalmol 1972;26:385-93.

8. Hunter GD, Repka MX. Diode laser photocoagulation for threeshold retinopathy of prematurity. A randomized study. Ophthalmology 1993;100:238-44.

9. McNamara JA, Tasman W, Brown GC, Federman JL. Laser photocoagulation for stage 3+ retinopathy of prematurity. Ophthalmology 1991;98:576-80.

10. Moraes NSB, Farah ME, Bonomo PP, Almeida MFB. Laser de diodo versus crioterapia no tratamento da retinopatia da prematuridade: estudo comparativo. Arq Bras Oftalmol 1997;60:635-8

11. Capone A, Diaz-Rohena R, Sternberg P, Mandell B, Lambert HM, Lopez PF. Diode-laser photocoagulation for zone 1 threshold retinopathy of prematurity. Am J Ophthalmol 1993;116:440-50.

12. McNamara JA. Laser treatment for retinopathy of prematurity. Cur Opin Ophthalmol 1993;4:76-80. 